EPJ manuscript No.

(will be inserted by the editor)

\title{
Classical and quantum effects in spatially modulated optical parametric oscillators
}

\author{
Maria M. de Castro $^{1}$, Damià Gomila ${ }^{1}$, and Roberta Zambrini ${ }^{1, a}$ \\ Institute for Cross-Disciplinary Physics and Complex Systems, IFISC (CSIC-UIB), Palma \\ de Mallorca, 07122, Spain.
}

\begin{abstract}
We study the effects of transverse spatial modulations in a multimode degenerate optical parametric oscillator. Intracavity photonic crystals allow us to tune the instability threshold and improve entanglement above threshold. Here we compare such results with the case in which the modulation is in the injected field profile.
\end{abstract}

\section{Introduction}

Photonic crystals (PC) are materials with regularly alternating regions of high and low dielectric constant $\varepsilon$ or refractive index $n$ ( $n^{2} \sim \varepsilon$ for most optical purposes). A periodicity in $n$ can generate gaps at some frequencies for traveling electromagnetic waves. Analogous to the case of semi-conductors, which prevent electrons from passing through under some conditions, a $\mathrm{PC}$ with a wavenumber $k_{P C}$ can prevent the propagation of waves with a wavenumber $k=k_{P C} / 2[1,2]$.

The interest in photonic crystals stems from their capability of controlling light in ways that is not possible with conventional optics. Beyond their extraordinary properties for guiding, PC can also be used to engineer the transverse properties of the light. For instance, unwanted transverse spatial instabilities, that often spoil the quality of the beams in broad area nonlinear optical devices, can be controlled by an intracavity photonic crystal with a modulation in the transverse direction. The inhibition of such higher order transverse modes was first proposed theoretically in [3] and later observed experimentally in [4] and [5]. Roughly speaking, the inhibition of spatial instabilities in the transverse plane takes place because the unstable transverse modes fall within the band-gap of the photonic crystal [3]. This would suggest the inhibition of spontaneous pattern formation to be a rather general phenomenon and, in fact, it was observed either in the case of cubic or of quadratic nonlinearity [6].

On the other hand, effects of the interplay between translational symmetry breaking due to a PC and modulation instability can be rather complex. An example of a different scenario is the case in which two light fields at different frequencies are nonlinearly coupled, as in optical parametric oscillators (OPOs). These devices can present pattern emission at the down-conversion threshold, so that the homogeneous pump beam at frequency $2 \omega$ generates a transversally spatially modulated field at frequency $\omega$. If both fields at $2 \omega$ and $\omega$ are resonant in the cavity, the (spatial) instability can be either inhibited or stimulated by means of PCs modulations in the

a e-mail: roberta@ifisc.uib-csic.es 
transverse plane [7]. Furthermore, in Ref. [8] it was experimentally shown that the modulation instability in a nonlinear single feedback system with a photonic lattice can be either enhanced or suppressed depending on the lattice strength. Recently, these fundamental ideas about the role of spatial modulations at wavelength similar to spatial instability where also explored in other systems [9].

Beside these classical effects, in Ref. [7] we predicted the possibility of controlling spatial quantum fluctuations and improving Einstein-Podolsky-Rosen (EPR) entanglement [10] in an OPO with intracavity PC, in the following called PCOPO system. Here the multimode character of the OPO allows not only to investigate the effects of the PC on the spatial instability but also to establish if due to the PC band-gap, quantum noise in some spatial modes is suppressed leading to improved quantum effects. In the last years, spatial quantum correlations $[11,12]$ have been studied in different devices. The homogeneous multimode OPO was shown to present squeezing, entanglement and twin beams correlations between spatial modes below threshold and also above threshold, in presence of stable patterns or even frozen chaos [13,14]. Spatial quantum effects have been also predicted in Kerr media [15] and in second harmonic generation [16] and in the last years there have been several successful experimental realizations $[17-25]$.

Once established that quantum effects in the PCOPOs are deeply influenced by the presence of a spatial modulation due to a PC [7], the open question remains about the robustness of this phenomenon when spatially modulating the system in other ways. The aim of the present work is to compare the PCOPO with the OPO in which the modulation is imprinted in the transverse profile of the injected field. This comparison in different experimental configurations allows to generalize our results [7] establishing the role of breaking the translational symmetry by a modulation similar to the spontaneously emerging pattern, independently on the specific way in which this is realized (by pattern injection or detuning modulation). Pattern injection for classical light beams was considered in Ref. [26], or created in the input through a tilted-wave mechanism, as in Ref. [27].

In the following we introduce the model, Sec. 2, and analyze both classical effects on the instability process, Sec. 3, as well as quantum effects such as EPR entanglement between spatial modes, Sec. 4 .

\section{Model description}

By means of the quasi-probability representation formalism it is possible to model type I degenerate OPO with stochastic nonlinear partial derivative equations [1114,28,29]. As discussed in Ref. [14], in the Q-representation, the evolution of pump $\alpha_{0}(x, t)$ and down-converted signal $\alpha_{1}(x, t)$ slowly varying fields envelopes is governed by

$$
\begin{array}{r}
\partial_{t} \alpha_{0}(x, t)=-\left[\left(1+i \Delta_{0}\right)-i \nabla^{2}\right] \alpha_{0}(\mathbf{x}, t)+E-\frac{1}{2} \alpha_{1}^{2}(x, t)+\sqrt{\frac{2}{a}} \frac{g}{\gamma} \xi_{0}(x, t) \\
\partial_{t} \alpha_{1}(x, t)=-\left[\left(1+i \Delta_{1}\right)-2 i \nabla^{2}\right] \alpha_{1}(x, t)+\alpha_{0}(x, t) \alpha_{1}^{*}(x, t)+\sqrt{\frac{2}{a}} \frac{g}{\gamma} \xi_{1}(x, t),
\end{array}
$$

where time $t$ and transverse spatial direction $x$ are adimensional, being scaled with the decay time $\gamma$ and with the diffusion coefficient $a$, while $g$ is proportional to the nonlinear susceptibility [14]. These equations are valid for the device operating both below and above threshold, as far as $\left|\alpha_{0}(x, t)\right|<2$ that is for pump field below twice the modulus of the stationary field at threshold. Otherwise the dynamics of the 
Q representation would suffers from negative diffusion. Furthermore, as usual, this model description is valid within the mean field approximation and in the thin crystal limit, where deviations from free space diffraction can be neglected $[3,6,11-14,28-$ $30] . \xi_{0}(x, t)$ and $\xi_{1}(x, t)$ are additive and phase-sensitive multiplicative white noises respectively. In particular

$$
\left\langle\xi_{0}(x, t) \xi_{0}^{*}\left(x^{\prime}, t^{\prime}\right)\right\rangle=\delta\left(x-x^{\prime}\right) \delta\left(t-t^{\prime}\right)
$$

while

$$
\xi_{1}(x, t)=\left[\frac{-\alpha_{0 I}(x, t)}{2 \sqrt{2+\alpha_{0 R}(x, t)}}+\frac{i}{2} \sqrt{2+\alpha_{0 R}(x, t)}\right] \phi(x, t)+\sqrt{\frac{1-\frac{\left|\alpha_{0}(x, t)\right|^{2}}{4}}{2+\alpha_{0 R}(x, t)}} \psi(x, t)
$$

with $\alpha_{0}=\alpha_{0 R}+i \alpha_{0 I}$ and $\phi, \psi$ real:

$$
\begin{aligned}
\left\langle\phi(x, t) \phi\left(x^{\prime}, t^{\prime}\right)\right\rangle & =\delta\left(x-x^{\prime}\right) \delta\left(t-t^{\prime}\right) \\
\left\langle\psi(x, t) \psi\left(x^{\prime}, t^{\prime}\right)\right\rangle & =\delta\left(x-x^{\prime}\right) \delta\left(t-t^{\prime}\right) .
\end{aligned}
$$

The other parameters are pump and signal detunings $\Delta_{0,1}$ and the input pump field $E$. For negative signal detuning, the parametric threshold of the oscillator coincides with a spatial instability threshold [30] leading to signal emission presenting a transverse stripe pattern at wave-number $k_{c}=\sqrt{-\Delta_{1} / 2}$. Without loss of generality we stand $\Delta_{0}=0$ and $\Delta_{1}=-1$.

We consider now two different variations of this model. The first one is a PCOPO, already presented in Ref. [7] where a sketch of the experimental set-up is shown. Here we focus on the case in which the pump detuning is spatially modulated so that Eq. (1) is replaced by

$$
\begin{array}{r}
\partial_{t} \alpha_{0}(x, t)=-\left[\left(1+i\left(\Delta_{0}+M_{0} \sin \left(k_{P C} x\right)\right)\right)-i \nabla^{2}\right] \alpha_{0}(\mathbf{x}, t) \\
+E-\frac{1}{2} \alpha_{1}^{2}(x, t)+\sqrt{\frac{2}{a}} \frac{g}{\gamma} \xi_{0}(x, t)
\end{array}
$$

with spatially modulated detuning whose amplitude is $M_{0}$ and wave-number $k_{P C}$. As expected, the most interesting situation corresponds to the case in which the spatial instability takes place in the photonic band-gap [3,6,7]. Similarly to our analysis in Ref. [7], being the OPO emission characterized by a signal with critical wave number $k_{c}$, we restrict ourselves to the case $k_{P C}=2 k_{c}$. Due to the nonlinear coupling between pump and signal light beams, the spatial periodicity in the pump is characterized by $k_{\text {pump }}=2 k_{\text {signal }}[30]$. Therefore in this case the PC and pattern in the pump field have the same periodicity [7].

The second scheme corresponds to an OPO where, instead of a homogeneous pump field, we consider an injected pattern. The dynamics of this injected pattern OPO (IPOPO) is governed by

$$
\begin{gathered}
\partial_{t} \alpha_{0}(x, t)=-\left[\left(1+i \Delta_{0}\right)-i \nabla^{2}\right] \alpha_{0}(x, t)+ \\
+E+E_{0} \sin k_{P C} x-\frac{1}{2} \alpha_{1}^{2}(x, t)+\sqrt{\frac{2}{a}} \frac{g}{\gamma} \xi_{0}(x, t)
\end{gathered}
$$

together with Eq. (2). In the following, for the sake of comparison, we consider a pattern injected with the same transverse modulation than the detuning of the former $\mathrm{PCOPO}$ case $k_{P C}=2 k_{c}$. 


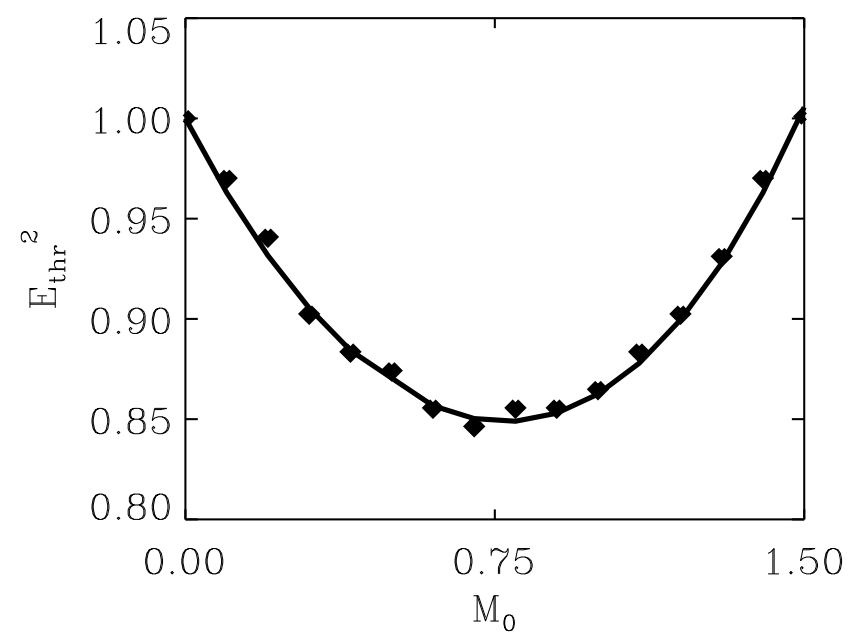

Fig. 1. PCOPO instability threshold as a function of the amplitude modulation of the pump detuning. Dots are numerical results and the continuous line is obtained from the linear stability analysis of Eqs. (7) amd (2).

\section{Tuning thresholds}

In Ref. [7] it was shown that an intracavity PC with appropriate periodicity in an OPO can increase or lower the threshold for the emission of the signal field. Essentially, if a PC with a wavenumber $k_{P C}=2 k_{c}$ modulates signal detuning, one can inhibit the off-axis emission, increasing the threshold. This is the pattern inhibition expected due to the band-gap $[3,6]$. On the other hand, if a PC with the same periodicity affects the pump field $\left(M_{0} \neq 0\right)$, the threshold can be lowered because the PC reinforces the modulation of the pump above threshold (Figs. 1 and 2a). This reduction is potentially useful since less energy is needed to achieve signal emission, translating into a better efficiency of the device [7].

One could also expect a similar effect by modulating the pump at the input $\left(E_{0} \neq 0\right)$, however, this is not exactly the case. Fig. 2 shows the intensity of the signal field at critical wave-number as a function on the incoming homogeneous pump for different values of $E_{0}$ and $M_{0}$. The same bifurcation than in an OPO is observed in both PCOPO and IPOPO, i.e. the response of the system upon modulation of the homogeneous pump is similar to the case where the modulation comes from a PC. The signal is zero below a certain threshold and above its intensity grows linearly with the input pump. Futhermore, looking at the instability diagrams in Figs. 1 and $3 \mathrm{a}$, a reduction of the threshold in the amplitude of the homogeneous pump $E$ is found for small values of modulations $M_{0}$ and $E_{0}$, respectively. On the other hand, at difference with the case of the modulation introduced by a PC, here this effect does not imply a better efficiency of the device, since including a modulation in the input field has an extra cost in terms of energy. Fig. 3b) shows the energy introduced in the system $U$ at threshold as a function of the amplitude of the modulation $E_{0}$. We observe a small reduction for small amplitudes of the perturbation $E_{0}$ but, for values of $E_{0}>0.5$, more energy has to be introduced in the system to achieve signal emission. Therefore we conclude that a modulation of the input field does not have the same effect of an intracavity PC modulating the detuning. As a matter of fact when considering the input energy, pattern injection is not a suitable way to reduce the threshold for signal emission in OPO's. 

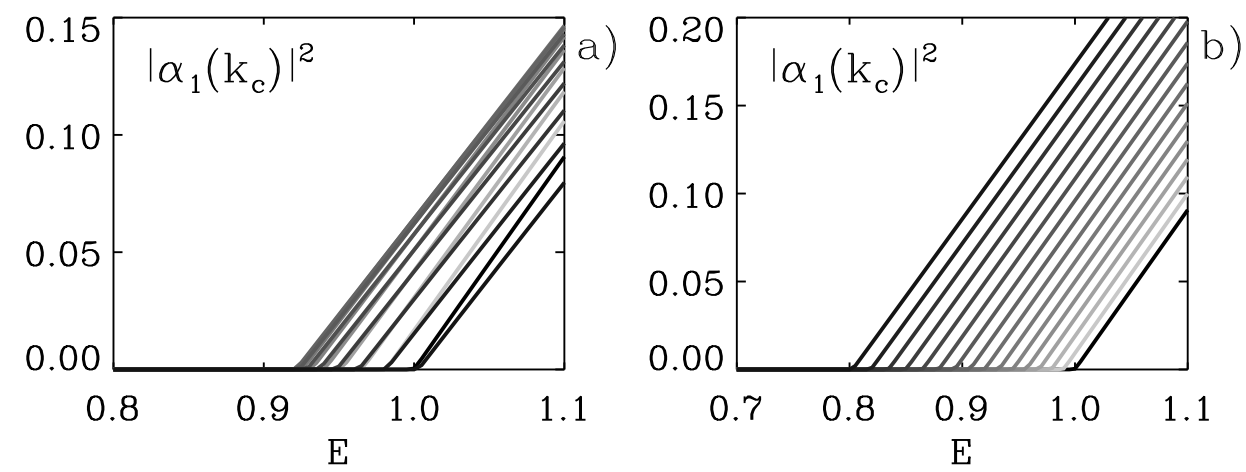

Fig. 2. Bifurcation diagram for the parametric down conversion process in PCOPO (a) and in a IPOPO (b). Different values of $M_{0}$ in the interval $[0,1.5]$ (a) and $E_{0}$ in the interval $[0,1]$ (b) are represented in gray scale, the lighter the color the smaller the modulation, being in black the OPO case.
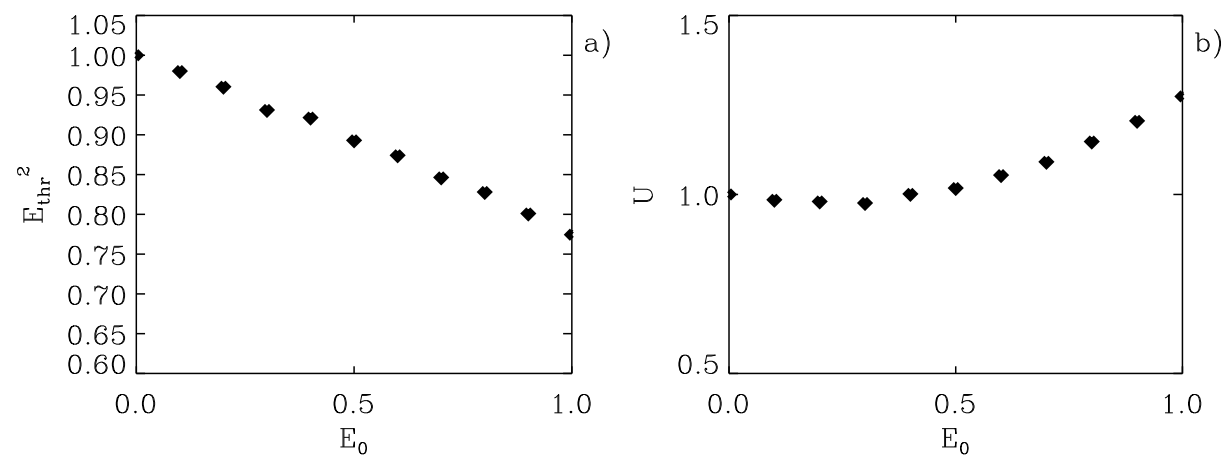

Fig. 3. Numerical results for the instability threshold as a function of the amplitude of the modulated injected pump. In b) instead of the intensity of the homogeneous pump we plot the total pumped energy $U=E_{t h r}^{2}+\left(E_{0}^{2} / L\right) \int_{0}^{L} d x \sin ^{2} k_{P C} x$ at threshold, being $L$ the transverse size of the system.

\section{EPR entanglement}

The interplay between nonlinearity and spatial coupling (through diffraction) gives rise not only to a variety of regimes for the spatio-temporal dynamics, but also to correlations between different modes of the light beam. In this Section we aim to establish the effect of a modulation in the transverse plane on spatial entanglement. Spatial entanglement in OPO was already considered in different configurations and regimes $[13,29]$. In Ref.[7] we presented evidence of the beneficial effect of an intracavity photonic crystal in order to get spatial entanglement in OPO operating above threshold. Here we will compare this device in the two configurations in which the input light beam is modulated (IPOPO) or an intracavity photonic crystal is included (PCOPO), both above and below the respective thresholds.

As usual [29], in order to study EPR entanglement between opposite far field modes $(k$ and $-k)$, we consider the quadratures superposition operator

$$
\begin{array}{r}
\Sigma_{\theta \phi}(k,-k)=\left(\hat{A}(k)+\hat{A}(-k) e^{i \phi}\right) e^{i \theta}+\left(\hat{A}^{\dagger}(k)+\hat{A}^{\dagger}(-k) e^{-i \phi}\right) e^{-i \theta}= \\
=\left(\hat{A}(k) e^{i \theta}+\hat{A}^{\dagger}(k) e^{-i \theta}\right)+\left(\hat{A}(-k) e^{i(\theta+\phi)}+\hat{A}^{\dagger}(-k) e^{-i(\theta+\phi)}\right) .
\end{array}
$$


with superposition angle $\phi$ and quadrature angle $\theta$. The analogy with the EPR paradox [10] is obtained by introducing the positions sum and momenta difference of the two modes, given by

$$
\hat{x}_{1}+\hat{x}_{2}=\hat{A}(k) e^{i \theta}+\hat{A}(-k) e^{i(\theta+\phi)}+\text { h.c. }=\Sigma_{\theta \phi}(k,-k)
$$

and by

$$
\hat{p}_{1}-\hat{p}_{2}=\frac{1}{i}\left(\hat{A}(k) e^{i \theta}-\hat{A}(-k) e^{i(\theta+\phi)}\right)+h . c .=\Sigma_{\theta^{\prime} \phi^{\prime}}(k,-k) .
$$

where the latter corresponds to a shift $\theta \rightarrow \theta^{\prime}=\theta-\pi / 2$ and $\phi \rightarrow \phi^{\prime}=\phi+\pi$ and subscripts 1 and 2 refer to two far field spatial modes $\pm k$.

A well-known criterion for EPR entanglement proposed by Reid and Drummond [31] establishes a lower bound for the variances product below which the state of the system is entangled. In presence of violation of this inequality there are two non-commuting observables (say $x_{1}$ and $p_{1}$ ) strongly correlated to the corresponding variables in another subsystem (say $x_{2}$ and $p_{2}$ ) so that measurement of either observable in one of the two sub-systems allows the inference of that variable in the other one, with an accuracy better than the standard quantum limit. We notice that the best inference is obtained considering conditional variances. This is obtained by minimization over a parameter $\lambda$ of the variance of $\Sigma_{\theta \phi}^{\lambda}=\hat{x}_{1}+\lambda x_{2}$ leading to $\lambda=\frac{-\left\langle\hat{x}_{1} \hat{x}_{2}\right\rangle+\left\langle\hat{x}_{1}\right\rangle\left\langle\hat{x}_{2}\right\rangle}{\Delta^{2} \hat{x}_{2}}$. The criterion [31] then establishes that the spatial modes are EPR entangled if

$$
\Delta^{2} \Sigma_{\theta_{0}, \phi_{0}}^{\lambda} \Delta^{2} \Sigma_{\theta_{0}+\pi / 2, \phi_{0}+\pi}^{\lambda} \leq 1
$$

for some angles $\theta_{0}, \phi_{0}$. In the following we calculate EPR entanglement focusing on the signal field $\hat{A}_{1}$ in its most intense far field modes, appearing at the critical wavenumber $k_{c}=\sqrt{-\Delta_{1} / 2}$. The operator (9) is then considered for $\hat{A}_{1}\left( \pm k_{c}\right)$ and its variance is numerically calculated by simulation of the equations presented in Sect.2. In order to establish the presence of EPR entanglement, Eqs.(7-2) (for PCOPO) as well as Eqs.(8-2) (for IPOPO) are numerically simulated considering all spatial modes, obtaining first and second order moments of $\alpha_{1}\left( \pm k_{c}\right)$ in the antinormal ordering, corresponding to the Husimi representation. After some manipulations [32], the normally ordered output moments [33] are obtained. An alternative approach is reported in Ref. [32] where analytical expressions for this quantities for a PCOPO below threshold are obtained based on an approximated (few modes and linear) description of the system.
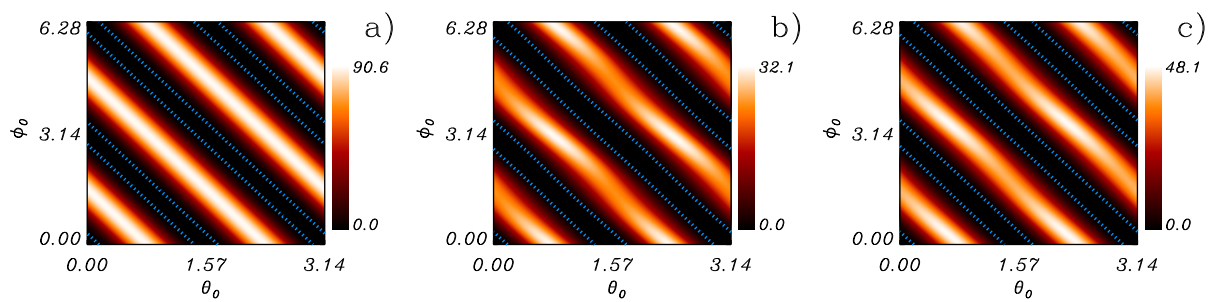

Fig. 4. (Color online) Product of the variances of $\Sigma_{\theta_{0}, \phi_{0}}$ and $\Sigma_{\theta_{0}+\pi / 2, \phi_{0}+\pi}$ as a function of the superposition $\phi_{0}$ and quadrature $\theta_{0}$ angles, $10 \%$ below the corresponding threshold. Blue dashed lines limit regions with spatial EPR entanglement for an OPO (a), a PCOPO (b), and a IPOPO (c).

It is well known that spatial entanglement is found below threshold in OPOs [29] and that this phenomenon is sensitive to the distance form the threshold. As shown 
in the previous Section, spatial modulations either in the incoming field or in the pump detuning have the effect of changing the instability threshold. Therefore spatial entanglement will be deeply modified in both configurations considered here with respect to the OPO: for a fixed pump $E$ both squeezing and entanglement improve when the threshold is lowered, worsening for pattern inhibition (higher threshold). On the other hand, if we compare OPO and PCOPO (with intracavity PC) at the same distance from the threshold, there is not substantial difference in quadrature squeezing and entanglement, as shown in Ref. [7]. In Fig. 4 we present numerical results $10 \%$ below the corresponding thresholds for the OPO, PCOPO and IPOPO, showing that angular regions of entanglement are preserved both in presence of $\mathrm{PC}$ and pattern injection. We then conclude that spatial modulations influence the instability threshold preserving spatial quantum effects below threshold, being the distance from the instability regime the only relevant parameter.
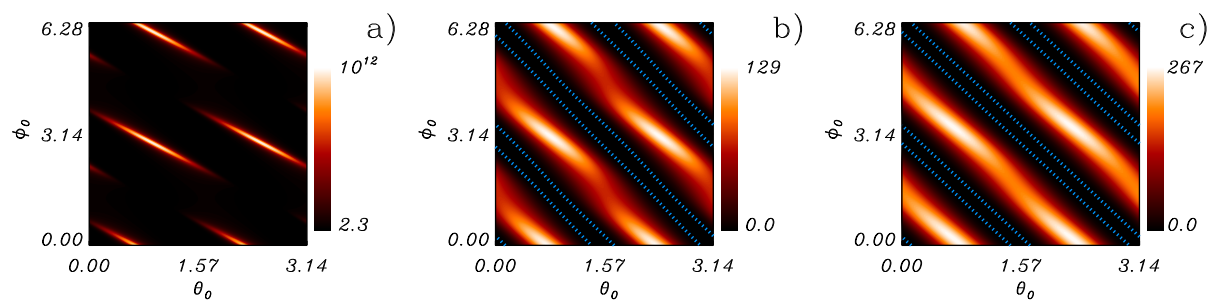

Fig. 5. (Color online)(Color online) Product of the variances of $\Sigma_{\theta_{0}, \phi_{0}}$ and $\Sigma_{\theta_{0}+\pi / 2, \phi_{0}+\pi}$ as a function of the superposition $\phi_{0}$ and quadrature $\theta_{0}$ angles, $2 \%$ above the corresponding threshold. Blue dashed lines limit regions with spatial EPR entanglement for an OPO (a), a PCOPO (b), and a IPOPO (c).

We now move to the above threshold regime, Fig. 5. In this case, as shown in Ref. [7], an intracavity PC does have beneficial effects on EPR entanglement. This was explained as an effect of the break of translational symmetry into the system due to the transverse PC modulation. In fact, the position of the stripe pattern arising above threshold is locked by the PC. This has the effect of strongly reducing phase fluctuations leading to EPR entanglement also above threshold. In Fig. 5 we compare the OPO withe both PCOPO and IPOPO. In both configurations with spatial modulations there are large angular areas where correlations are below the classical level (blue dashed lines in the figures) meaning that entanglement is found in a rather large range of quadratures. Therefore both pattern injection and detuning modulation improve EPR entanglement above threshold.

\section{Conclusion}

We have compared OPO, PCOPO (with detuning modulation for the pump field) and IPOPO (with injected pattern) focusing on their instability threshold and quantum correlations. Both pattern injection and pump detuning modulation influence the instability process. In both cases there is a competition between pattern stimulation (because the pump pattern is seeded in the system) and pattern inhibition (due to the fact that the signal at the critical mode is in the band-gap for $k_{P C}=2 k_{c}$ ). At first sight this competition leads to reduced instability thresholds but, once considering both PCOPO and IPOPO schemes for the same input energy, only the PCOPO 
shows significant threshold lowering, while pattern injection is mostly inhibiting the instability.

We have then characterized quantum fluctuations, focusing here on EPR spatial entanglement following the Reid-Drummond criterion [31] both below, Fig. 4, and above threshold, Fig. 5. We find that both PCOPO and IPOPO display similar entanglement than the OPO at the same distance below threshold while above threshold both schemes have a similar improvement with respect to the OPO. The reason of the similarity of these schemes when considering quantum effects at a fixed distance from the threshold may be interpreted as follows. Both detuning modulation and pattern injection couple different spatial pump modes. In the PCOPO, detuning modulation leads to hopping between the $k=0$ mode and the shifted ones at $\pm k_{P C}$ in the pump field. In the IPOPO the same tilted modes $\pm k_{P C}$ (together with the homogeneous one) are injected into the system in the pump field. In both cases, once considered the same distance from the threshold, there is an incoherent and similar down-conversion of these pump tilted modes $\pm k_{P C}$ into the signal ones $\pm k_{c}$ [34]. For this reason the effect of modulation in PCOPO and IPOPO on the correlations between the twin signal modes $\left( \pm k_{c}\right)$ is similar. On the other hand, the beneficial effect on entanglement found above threshold is mainly due to the pattern phase locking and to a related reduction of phase diffusion [15]. These results suggest that breaking translational symmetry is rather effective independently on the specific scheme considered. These predictions may have applications in quantum imaging, metrology, and quantum in-

formation and suggest a control mechanism of fluctuations by spatial modulation of interest also in other nonlinear systems [9].

R.Z. would like to dedicate this paper to Professor Federico Casagrande who introduced her into the fields of Nonlinear and Quantum Optics.

This work is supported by FISICOS (FIS2007-60327), ECuSCo (200850I047) and CoQuSys (200450E566) projects.

\section{References}

1. E. Yablonovitch, Phys. Rev. Lett. 58, (1987) 2059; S. John, Phys. Rev. Lett. 58, (1987) 2486.

2. J. D. Joannopoulos, S. G. Johnson, J. N. Winn, and R. D. Meade, Photonic Crystals: Molding the Flow of Light, 2nd edition (Princeton University Press, 2008)

3. D. Gomila, R. Zambrini and G-L. Oppo, Phys. Rev. Letters 92, (2004) 253904.

4. N. Marsal, D. Wolfersberger, M. Sciamanna, G. Montemezzani and D. N. Neshev. Optics Letters 33, (2008) 21.

5. B. Terhalle, N. Radwell, P. Rose, C. Denz and T. Ackemann, Applied Physics Letters 93, (2008) 151114

6. Damià Gomila and G.-L. Oppo, Phys. Rev. E 72, (2005) 016614.

7. M. M. de Castro, M. A. Garcia-March, D. Gomila, and R. Zambrini, Phys. Rev. A 84, (2011) 033832.

8. A.A. Sukhorukov, N. Marsal, A. Minovich, et al. Optics Letters 35, (2010) 3568; N. Marsal, D. Wolfersberger, M. Sciamanna et al. IEEE Journal of Quantum Elecronics 45, (2009) 1380.

9. A. V. Gorbach and D. V. Skryabin, Physical Rev. B 82, (2010) 125313; Yu. V. Bludov and V. V. Konotop, Phys. Rev. A 81, (2010) 013625; M. Odin Soler Rus, E. Cabrera-Granado and J.M. Guerra Perez, Physical Review A 83 (2011) 053824.

10. A. Einstein, B. Podolsky, and R. Rosen, Phys. Rev. 47, (1935) 777.

11. M. I. Kolobov, Rev. Mod. Phys. 71, (1999) 1539.

12. M. Kolobov (editor) Quantum Imaging (Springer, New York, 2007).

13. A. Gatti, H. Wiedemann, L. A. Lugiato, I. Marzoli, G.-L. Oppo, and S. M. Barnett, Phys. Rev. A 56, (1997) 877; R. Zambrini, S.M. Barnett, P. Colet and M. San Miguel, 
Physical Review A, 65, (2002) 023813; I. Perez-Arjona, E. Roldan, G.J. de Valcarcel, European Physical Letters 74, (2006) 247; M. Lassen, G. Leuchs, and U. L. Andersen, Phys. Rev. Lett. 102, (2009) 163602; B. Chalopin, F. Scazza, C. Fabre, and N. Treps, Phys. Rev. A 81, (2010) 061804(R).

14. R. Zambrini, S.M. Barnett, P. Colet and M. San Miguel, Eur. Phys. Journ. D 22, (2003) 461.

15. R. Zambrini, M. Hoyuelos, A. Gatti, P. Colet, L. Lugiato and M. San Miguel, Phys. Rev. A 62, (200) 063801.

16. M. Bache, P. Scotto, R. Zambrini, M. San Miguel, and M. Saffman Phys. Rev. A 66, (2002) 013809.

17. N. Treps, N. Grosse, W. P. Bowen, C. Fabre, H.-A. Bachor, and P.K. Lam, Science 301, (2003) 940.

18. V. Boyer, A. M. Marino, and P. D. Lett, Phys. Rev. Lett. 100, (2008) 143601.

19. V. Boyer, A.M. Marino, R.C. Pooser and P.D. Lett, Science, 321, (2008) 544.

20. A. M. Marino, R. C. Pooser, V. Boyer and P. D. Lett, Nature 457, (2009) 859.

21. J. Janousek, K. Wagner, J.-F. Morizur, N. Treps, P. K. Lam, C. C. Harb and H-A. Bachor, Nature Photonics 3 (2009) 399.

22. G. Brida, M. Genovese and I. Ruo Berchera, Nature Photonics 4, (2010) 227.

23. B. Chalopin, F. Scazza, C. Fabre and N. Treps, Phys. Rev. A 81, (2010) 061804(R).

24. M. Lassen, G. Leuchs, and U. L. Andersen Phys. Rev. Lett. 102, (2009) 163602.

25. B. Chalopin, F. Scazza, C. Fabre, and N. Treps, Optics Express, 19, (2011) 4405.

26. R. Martin, A.J. Scroggie, G.-L. Oppo, and W.J.Firth, Phys. Rev. Lett. 77, (1996) 4007.

27. W.J. Firth and A.J. Scroggie, European. Physical Letters 26, (1994) 521.

28. L. A. Lugiato, M. Brambilla, and A. Gatti, Advances in Atomic, Molecular and Optical Physics 40, 229-306, B. Bederson and H. Walther eds. Academic Press, New York, (1999).

29. A. Gatti, L.A. Lugiato, K.I. Petsas, I. Marzoli, European Physical Letters 46, (1999) 461; L. Lopez, B. Chalopin, A. Rivire de la Souchre, C. Fabre, A. Matre, and N. Treps, Phys. Rev. A 80, (2009) 043816; A. Gatti, K.I. Petsas, I. Marzoli and L.A. Lugiato, Optics Communications, 179, (2000) 591; R. Zambrini, A. Gatti, L. Lugiato and M. San Miguel, Phys. Rev. A 68, (2003) 063809.

30. G.-L. Oppo, M. Brambilla, and L.A. Lugiato, Phys. Rev. A 49, (1994) 2028.

31. M.D. Reid and P.D. Drummond, Phys. Rev. Lett. 60, (1988) 2731.

32. M. A. García-March, M. M. de Castro, and R. Zambrini, in preparation.

33. M.J. Collett and C.W. Gardiner, Phys. Rev. A 30, (1984) 1386.

34. R. Zambrini and M. San Miguel, Physical Review A 66, (2002) 023807. 